\title{
Science of the Europa Lander Mission Concept
}

K. P. Hand ${ }^{1}$, Jet Propulsion Laboratory, California Institute of Technology; A. E. Murray, Desert Research Institute and University, Reno, NV; J. B. Garvin, NASA Goddard Spaceflight Center; W. B. Brinckerhoff, NASA Goddard Spaceflight Center; B. Christner, University of Florida; K. E. Edgett, Malin Space Science Systems; B. Ehlmann, California Institute of Technology; C. German, Woods Hole Oceanographic Institution; A. Hayes, Cornell University; T. Hoehler, NASA Ames Research Center; S. Hörst, Johns Hopkins University; J. Lunine, Cornell University; K. Nealson, University of Southern California; C. Paranicas, Johns Hopkins Applied Physics Laboratory; B.E. Schmidt, Georgia Institute of Technology; D.E. Smith, Massachusetts Institute of Technology; A. Rhoden, Southwest Research Institute; M. Russell, Jet Propulsion Laboratory, Caltech; A. Templeton, University of Colorado; P. Willis, Jet Propulsion Laboratory, Caltech; A. Yingst, Planetary Science Institute, C. B. Phillips, Jet Propulsion Laboratory, Caltech; M. L. Cable, Jet Propulsion Laboratory, Caltech; K. L. Craft, Johns Hopkins Applied Physics Laboratory, T. A. Nordheim, Jet Propulsion Laboratory, Caltech; J. Pitesky, Jet Propulsion Laboratory, Caltech; M. Cameron, Jet Propulsion Laboratory, Caltech; J. D. Hofgartner, Jet Propulsion Laboratory, Caltech; A. E. Hofmann, Jet Propulsion Laboratory, Caltech; J. Scully, Jet Propulsion Laboratory, Caltech; J. Kosberg, Jet Propulsion Laboratory, Caltech; J. Foster, Jet Propulsion Laboratory, Caltech; E. Klonicki, Jet Propulsion Laboratory, Caltech.

1khand@jpl.nasa.gov, 626-487-5379. This research was carried out at the Jet Propulsion Laboratory, California Institute of Technology, under a contract with the National Aeronautics and Space Administration (80NM0018D0004). The information presented about the Europa Lander mission concept is pre-decisional and is provided for planning and discussion purposes only.

Along with the Science Definition Team and pre-Project Science Team listed above, an additional 146 scientists joined as co-signers on this paper (full list available upon request). The demographics of the primary disciplines represented by the signatories of this paper are shown below. As can be seen, this mission concept engages and includes the interests of not just physicists and geologists, but also many biologists and chemists. The engineers are primarily represented by instrument developers who are working with scientists to develop instrumentation for the model payload. Information on subdisciplines (e.g., planetary science, astrobiology, microbiology, geobiology, oceanography, etc.) is also available.
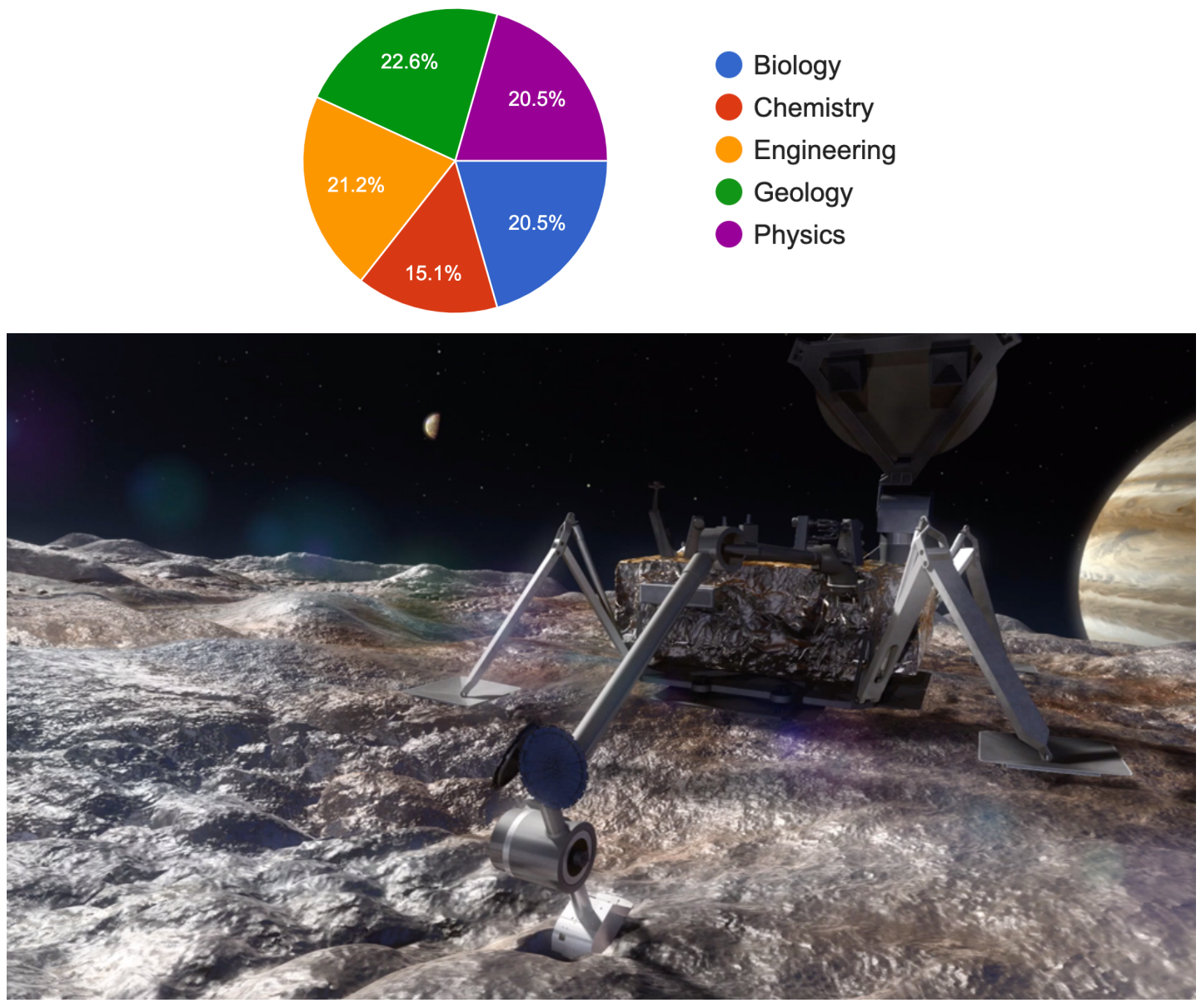

(C) 2020. California Institute of Technology. Government sponsorship acknowledged 


\section{Executive Summary}

Jupiter's moon Europa is a prime target in our exploration of potentially habitable worlds beyond Earth, and of oceans that likely exist beneath the icy shells of numerous worlds in the outer solar system. Europa may hold the clues to one of NASA's long-standing goals - to search for life elsewhere and determine whether or not we are alone in the universe (NASA, 2020) ${ }^{1}$.

The exploration of Europa presents an important target for both astrobiology and comparative oceanography, i.e., the opportunity to study liquid water oceans as a planetary process. Europa's icy shell also offers the opportunity to study tectonics and geologic cycles across a range of mechanisms (e.g., Earth's cooling versus Europa's tidal dissipation) and compositions (silicate in the case of the Earth, versus ice in the case of Europa).

Europa is a scientifically important and strategic target for both planetary science and astrobiology.

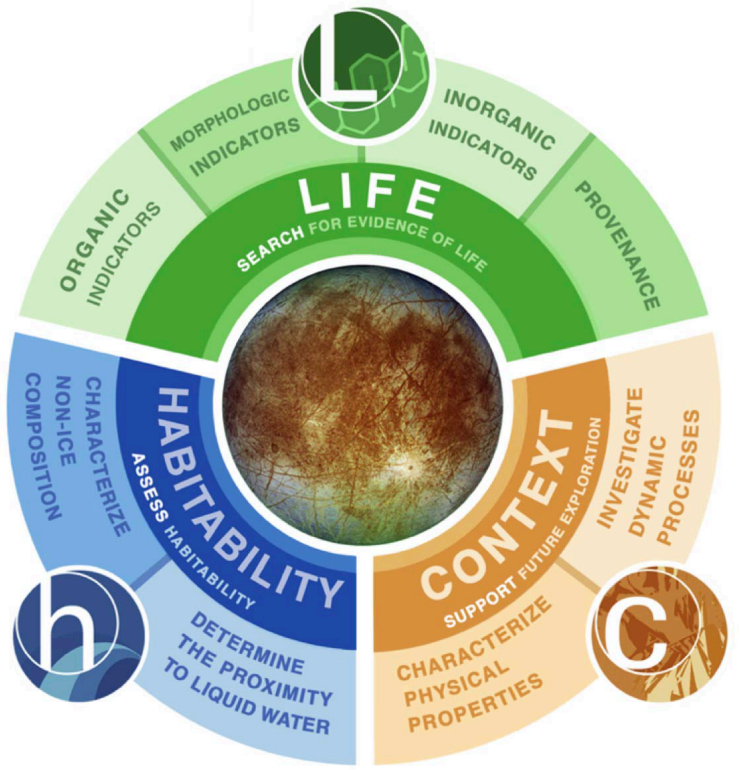

Figure 1. The science goals and objectives of the Europa Lander mission concept. These goals and objectives, as well as much of the technical design of the mission concept, are well-suited for landing and conducting science on many of the ocean worlds in our solar system.

Critically, Europa's subsurface ocean has likely existed for much of the history of the solar system, potentially providing a persistent, stable environment in which a second, independent origin of life may have arisen. Observations and models indicate that the ocean is likely in contact with a rocky, silicate seafloor, and the ice shell may have tectonic activity that could allow reductant-oxidant cycling. This scenario could lead to an ocean rich in the elements and energy needed for the emergence of life, and for potentially sustaining life through time. The persistence of Europa's ocean means that life could be alive there today - i.e., signs of extant life could be found within the ice and ocean of Europa. The discovery of signs of extant life is critical if we are to understand biology as a universal process: Does it contain DNA or does it function on some other large biomolecules for information storage, replication, and repair? Are there many separate 'trees of life' within our solar system, or is the tree of life on Earth the only one? The search for past life on worlds like Mars is very important, but the search for extant life is how we will truly revolutionize biology (if life exists beyond Earth).

Lander concepts for Europa have been studied for over two decades (JPL concept studies go back to 1997). In 2016 NASA convened a Science Definition Team (SDT) to develop the science, and mission concept, for a landed spacecraft that would achieve civilization-scale biosignature science, while also answering questions about the surface and subsurface environment. Figure 1 shows the high-level science goals and objectives of the mission concept, and Figure 2 shows the

\footnotetext{
${ }^{1}$ NASA (2020) https://science.nasa.gov/about-us/science-strategy. Please note that in the interest of saving space we have not included extensive references in this paper. We refer the reader to the exhaustive list found within the Europa Lander Science Definition Team Report (link in Section 2).
} 
mechanical configuration of the lander during surface operations. This mission is ready to move into Phase A.

The high-level science goals of the Europa Lander Mission Concept are:

1. Search for evidence of biosignatures on Europa.

2. Assess the habitability of Europa via in situ techniques uniquely available to a lander mission.

3. Characterize surface and subsurface properties at the scale of the lander to support future exploration.

These goals are achieved by employing a lander on the surface that collects and processes a minimum of three separate samples, each of at least seven cubic centimeter in volume, and acquired from a depth of at least $10 \mathrm{~cm}$.

\section{Overview}

Here we detail the science of the Europa Lander Mission Concept, but first we provide an overview of significant milestones and developments that may be of interest.

1. The mission concept passed its deltaMission Concept Review (dMCR) in November of 2018. The original Mission Concept Review (MCR) was held in June of 2017, and key feedback from the MCR board, and NASA HQ, was to reduce the mission cost by removing the carrier relay stage and instead use a Direct-to-Earth (DTE) communications link from the surface of Europa. The dMCR DTE concept was costed at $\$ 2.8 \mathrm{~B}$, in real-year dollars, for phases A-D. This was an Independent Cost Estimate, the details of which can be shared with the committee upon request. We can also provide the dMCR board review documents upon request.

2. The dMCR mission concept achieves high value science without requiring an excessive number of engineering 'miracles'; this mission aims to be the right 'first' mission to the surface of Europa and balances technical risk with science return and cost. Over the past decade, JPL and APL have examined a range of mission architectures - from minimal science ballistic probes and impactors, to highly capable melt probes. Ballistic probes initially appear 'simple' but detailed analyses reveal significant complexity for comparatively low science return. Melt probes and deep drills, meanwhile, achieve high-value science but require too many 'miracles', leading to high

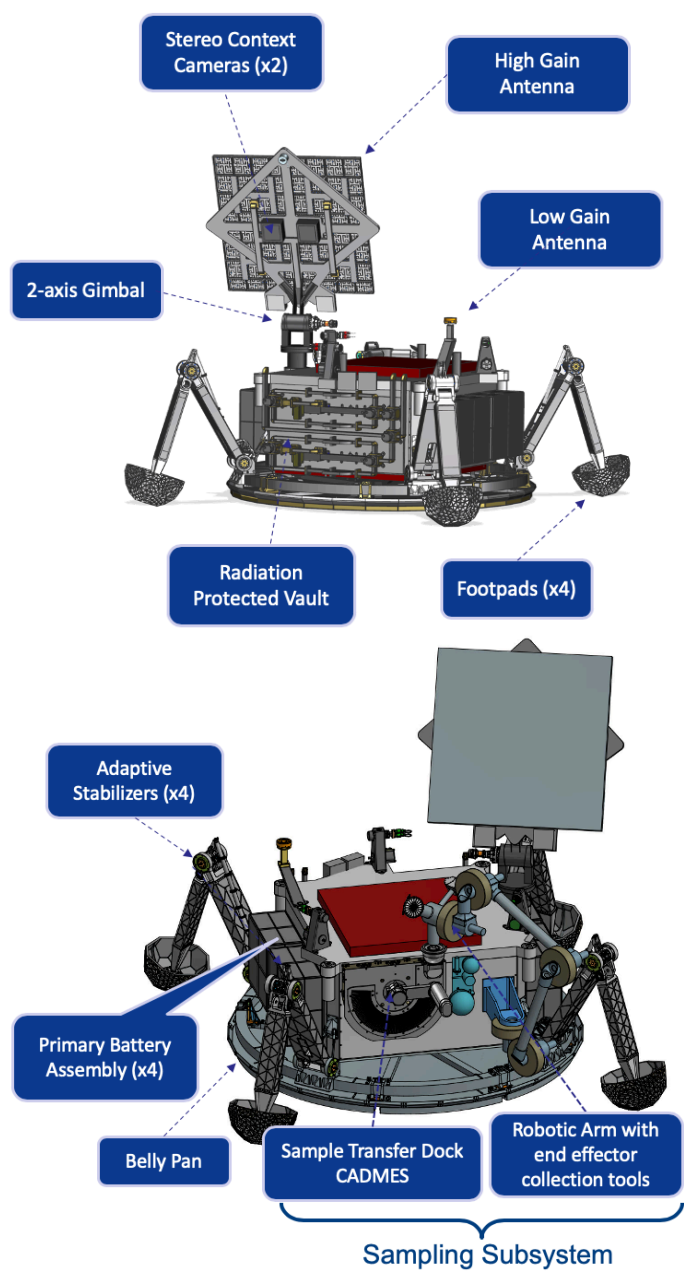

Figure 2. The Europa Lander concept (shown here in surface operations configuration) is mature and ready to move into Phase $A$. 
technical and cost risk. In addition, we have also examined options for lander missions that might fit into a Discovery or New Frontiers budget, but no viable options emerged.

3. The technology and instrumentation investments made to date (which exceed $\$ 300 \mathrm{M}$ ) could enable a new era of planetary exploration. Many of the technologies that have, or are, being developed for the Europa Lander Mission Concept can be utilized for landing on the unknown surfaces of many ocean worlds, and airless bodies in our solar system.

4. The Europa Lander builds on the investment in Europa Clipper, using data from that mission for landing site selection. There would be at least five years of time between the end of Clipper's prime mission and the landing site selection date. Importantly, data from Clipper would be unlikely to dramatically change our approach to de-orbit, descent, and landing (DDL). The mission concept team examined a variety of mechanical configurations and concluded that even after the acquisition of the Clipper data, the DDL and mechanical architectures would not significantly change. Uncertainty about parameters such as porosity and structure at the sub-meter scale would still require the intelligent landing system, with terrain relative navigation and hazard avoidance. Furthermore, the lander would still need to employ the 'snowshoe belly pan' and 'grasshopper' adaptive stabilizer legs to accommodate soft and variable surfaces at the submeter scale.

5. The Lander concept uses primary batteries and could survive for many weeks to $>60$ days on the surface, depending on sampling and idle power usage assumptions. The choice of primary batteries was, in part, to save on cost and complexity. A longer-lived mission concept with a radioisotope power system was studied, but planetary protection, thermal management, and mass were found to contribute to increasing cost and technical risk. The MCR and dMCR boards both determined that the surface lifetime from primary batteries was acceptable and helped limit planetary protection and cost risks.

6. In 2019, NASA selected 14 teams for development of instrumentation relevant to the Europa Lander model payload. The NASA ROSES "Instrument Concepts for Europa Exploration 2" (ICEE-2) program has helped advance many of the types of instruments needed for the in situ exploration of Europa and other ocean worlds. The ColdTech, SESAME, PICASSO, and MatISSE programs have also funded numerous technology and instrument developments to build a strong foundation for ocean worlds science investigations.

7. Below are links to several online resources related to this mission concept:

a. A full description of the science, including a full traceability matrix and traceability to past Decadal Surveys, can be found in the Science Definition Team report: https://europa.nasa.gov/resources/58/europa-lander-study-2016-report/

b. A video of the mission concept, which is based on a detailed trajectory, avionics, and mechanical framework, can be found here:

https://www.youtube.com/watch?v=pxin9qJVw48

c. A recording of our 2-hour virtual workshop, in which the mission concept, model payload, and sampling strategies were presented in detail, can be found here: https://www.europa-insitu.caltech.edu/

d. There are two Decadal Survey papers that focus on the technology and autonomy for this mission concept. The first authors of those papers are Ray Crum and Glenn Reeves, respectively. 


\section{Science Goals of the Europa Lander Mission Concept}

The high-level science goals of the Europa Lander Mission Concept were developed in ranked priority by the 2016 Science Definition Team, the full report of which can be found at the link above. The science addressed by the three Goals leads to a fully integrated mission concept, and model payload, that would enable a diverse approach to the search for potential biosignatures on an ocean world. Table 1 and Table 2 provide detail on the model payload and its relationship to the mission Goals and Objectives. The scientific and technical approach of the Europa Lander mission concept provides a robust, and in many ways conservative, strategy for the first landed mission to the surface of Europa.

\section{$\underline{\text { Science Goal \#1 is to Search for evidence of biosignatures on Europa. }}$}

The highest-level science goal of the Europa Lander mission is to search for evidence of biosignatures on Europa. The SDT leveraged the decades of work and experience from the Mars community and the biosignature framework that has helped guide the Mars program (Figure 3). The Lander payload measurements range from detecting and characterizing organic compounds, to looking for cell-like structures, to determining if the samples originate from within Europa's ocean (or other liquid water environments within the ice shell). The model payload enables detection and characterization of morphologic, chemical, and mineralogical indicators of life, all within a well-characterized geologic and geophysical context.

The organic chemical analyses are specifically targeted to reveal the broadest possible range of signatures produced by life, including analyses of molecular type, abundance, and chirality. Chemical analyses of samples collected directly from Europa's near surface layer would provide for characterization of organics at the picomole-per-gram level of sampled material, which is an improvement of approximately nine orders of magnitude over those possible by means of remote sensing capabilities. Spectroscopic analyses of samples provide the inorganic and geochemical context of the samples, and enable differentiation between Europa's endogenous chemistry and materials that may have been externally delivered (exogenous, e.g., from micrometeorites), or processed by Europa's radiation environment.

Finally, high-resolution imaging from lander instruments would span from microns to decameters, providing imagery for potential biosignatures as well as in situ context for sampled materials, local geology, and surface properties. This roughly seven orders of magnitude enhancement in spatial resolution over the Europa Clipper mission would provide key insights into the properties of Europa's ice shell, and any subsurface liquid water. Importantly, the model payload and measurements defined for Goal 1 generate highly valuable scientific results even in the absence of any signs of life.

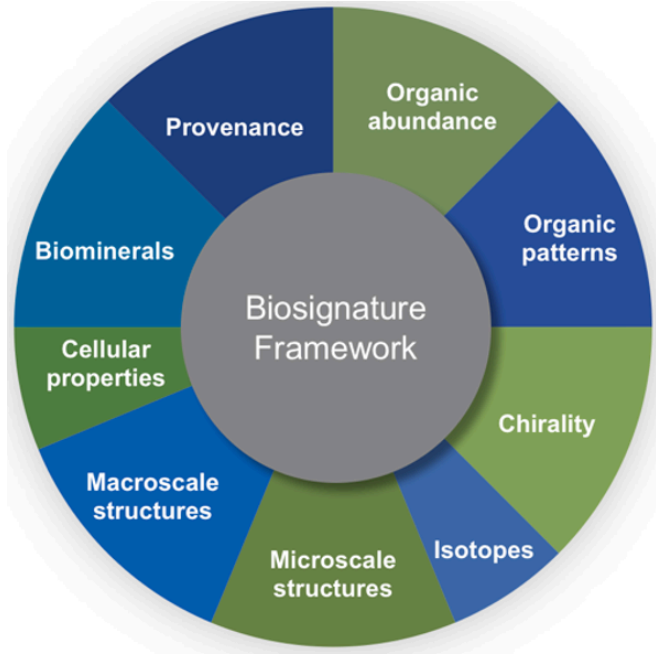

Figure 3. Biosignature framework showing the set of measurements that could be made on each sample collected by the Europa Lander. This robust set of biosignature measurements is highly complementary and redundant. 


\section{Science Goal \#2 is to assess the habitability of Europa via in situ techniques.}

Critically, the Europa Lander mission would advance our scientific understanding of fundamental aqueous and geochemical processes in the solar system, independent of whether or not signs of life are discovered on Europa. As part of this goal, measurements would be conducted that could 1) answer fundamental questions about the chemistry and geology of Europa's ice shell and ocean, and 2) provide ground-truth for Europa Clipper measurements.

Investigations of habitability include characterizing the non-ice composition of Europa's nearsubsurface to discern indicators of chemical disequilibria and other key environmental features that are essential to support life. This includes determining the abundances of $\mathrm{Cl}$-containing compounds, carbonates, sulfates, metal hydroxides, silica, and silicates, if present, at levels of a few to hundreds of parts per thousand by mass. Also critical is determining the abundances of volatiles such as $\mathrm{H}_{2} \mathrm{~S}$, $\mathrm{CH}_{4}, \mathrm{O}_{2}, \mathrm{H}_{2} \mathrm{O}_{2}, \mathrm{SO}_{2}, \mathrm{CO}_{2}, \mathrm{CO}, \mathrm{CH}_{3} \mathrm{SH}$, and dimethyl sulfide (DMS), if present, at levels of a few parts per thousand by mass. This collection of measurements could help determine the chemistry of Europa's ocean and the extent to which the ocean is cycling with a rocky seafloor and the overlying ice shell.

Goal 2 also includes geophysical (e.g., seismic) investigations to search for any subsurface liquid water within $30 \mathrm{~km}$ of the lander, including the ocean, and a search for evidence of interactions with liquid water on the surface at any scale (including active plumes and ejected materials). Depending on the extent of geologic activity and acoustic sources, Goal 2 may also enable the determination of the depth of Europa's ocean.

Acoustic sounding measurements would provide unique and highly complementary measurements to those performed by the radar, magnetometer, and plasma instruments which will be flown on Europa Clipper. The lander offers a highly-complementary approach in which one specific region could be monitored for activity over several tidal cycles with surface observations.

\section{Science Goal \#3 is to Characterize surface and subsurface properties at the scale of the} lander to support future exploration.

This goal would frame the measurements of the first two goals in the broader context of Europa as a potentially active and dynamic ocean world. Investigations made as part of this Goal include characterizing textural, structural, and compositional heterogeneities in surface and near-surface materials through measurements of the samples, and through observations of the terrain, from the lander workspace to the horizon and into the ice shell. The combination of these multi-scale measurements would aid in understanding the physical, chemical, and mechanical properties of the ice shell and any associated regolith, and would serve to characterize dynamic processes on and within Europa.

The measurements made as part of this goal could enable future exploration across Europa's surface, or deeper into its subsurface. The Europa Lander mission concept is a 'pathfinder' for the exploration of Europa, and potentially many other ocean worlds of the outer solar system. As with Goals 1 and 2, the measurements made as part of this goal would also extend and enhance the remote sensing and flyby in situ observations of the Europa Clipper Mission by performing surface to subsurface in situ analytical investigations of Europa's materials and ice shell, thereby providing valuable ground-truth measurements. 


\begin{tabular}{|c|c|c|}
\hline \multirow{2}{*}{$\begin{array}{c}\text { Instrument Class } \\
\text { [mass allocation, unmargined], } \\
\text { Total }=\mathbf{4 2 . 5} \mathrm{kg} \text { (with margin) }\end{array}$} & \multicolumn{2}{|c|}{ Model Payload } \\
\hline & Baseline & Threshold \\
\hline $\begin{array}{l}\text { Context Remote Sensing Instrument } \\
\text { (CRSI) [4.3 kg, includes shielding] }\end{array}$ & $\begin{array}{l}2 \text { identical multi-filter, focusable, visible to } \\
\text { near-infrared, stereo overlapping cameras with } \\
\text { narrowband filters equivalent to those of the } \\
\text { Europa Multiple Flyby Mission EIS cameras }\end{array}$ & $\begin{array}{l}2 \text { identical RGB, fixed focus, stereo } \\
\text { overlapping cameras }\end{array}$ \\
\hline $\begin{array}{l}\text { Microscope for Life Detection } \\
\text { (MLD) }\end{array}$ & \multirow{2}{*}{$\begin{array}{l}\text { Deep UV resonance Raman and optical } \\
\text { microscope with fluorescence spectrometer }\end{array}$} & $\begin{array}{l}\text { Atomic Force Microscope (AFM) } \\
\text { with optical context imager }\end{array}$ \\
\hline $\begin{array}{l}\text { Vibrational Spectrometer } \\
\text { (VS) } \\
\text { [V.4 kg] }\end{array}$ & & Raman Laser Spectrometer (RLS) \\
\hline $\begin{array}{l}\text { Organic Compositional Analyzer } \\
\text { (OCA) }\end{array}$ & $\begin{array}{l}\text { Gas Chromatograph Mass Spectrometer } \\
\text { (GC-MS) with Chirality Analysis and Stable } \\
\text { Isotope Analyzer (SIA) }\end{array}$ & $\begin{array}{l}\text { Gas Chromatograph Mass } \\
\text { Spectrometer (GC-MS) with } \\
\text { Chirality Analysis }\end{array}$ \\
\hline $\begin{array}{l}\text { Geophysical Sounding System } \\
\text { (GSS) }[1.2 \mathrm{~kg}]\end{array}$ & Broad-band seismometer & 3 -axis geophone \\
\hline
\end{tabular}

Table 1. Model payload and mass allocations for the Europa Lander mission concept. Shown at right are the Baseline and Threshold versions of the instruments. The instruments housed within the main body of the lander vault (which provides radiation shielding) are shown in white; elements outside the vault are shown in orange. The details and differences between Baseline and Threshold can be found in the full Science Definition Team report. A summary of the payload is provided in Section 4.

\begin{tabular}{|c|l|l|l|l|l|}
\hline \multicolumn{2}{|c|}{} & \multicolumn{2}{|c|}{ Notional } \\
Instruments
\end{tabular}

Table 2. Simplified version of the full Science Trace Matrix (STM) for the Europa Lander Mission concept. The full STM can be found in the SDT Report. Notional instruments are shaded dark blue for Objectives to which they are directly responsive, and light blue for a secondary or tertiary role. Instrument acronyms are provided in Table 1. LISS, shown in gray, refers to Lander Infrastructure Sensors for Science and refers to serendipitous science that could be achievable through the use of the landing and sampling systems on the spacecraft. 


\section{Model Payload}

For each science objective, model instruments are described in the SDT report. To demonstrate the overall scientific and technical viability of the Europa Lander mission concept, two example payload configurations (Baseline and Threshold) were developed in detail, based on flight-proven technologies that could be adapted to Europa conditions (Table 1). These example model payloads fit within the currently-established engineering constraints of the Europa Lander mission concept, and achieve the Baseline and Threshold level science requirements. All model payload instruments work in service to numerous goals, objectives, and investigations.

The mass allocation of $42.5 \mathrm{kgs}$ for the payload puts the lander well above the MER-class payload $(<8 \mathrm{~kg})$, but below that of MSL/Curiosity ( $\sim 65 \mathrm{kgs})$. With the exception of the Context Remote Sensing Instrument (CRSI), all instruments are held within a vault that provides radiation shielding. The centerpiece instruments for characterizing potential biosignatures are:

1) an Organic Compositional Analyzer (OCA), which in the Baseline model payload is a Gas Chromatograph-Mass Spectrometer (GC-MS) capable of achieving a 1 picomole per gram of sample limit of detection for organics,

2) a microscope system (referred to as the Microscope for Life Detection, MLD) capable of distinguishing microbial cells as small as $\mathbf{0 . 2}$ microns in diameter, and as dilute as 100 cells per cubic centimeter (cc, or equivalently $1 \mathrm{~mL}$ ) of ice. In the Baseline model payload this capability is to be addressed by a combination of spectroscopy and atomic force microscopy (AFM) or optical light microscopy (OM), and,

3) a Vibrational Spectrometer (VS), which in the Baseline model payload is a Raman and Deep UV fluorescence spectrometer capable of characterizing both organic and inorganic compounds down to a level of parts per thousand by mass.

The model payload also includes a pair of color stereo imagers (CRSI) for examining the landing, and a geophysical sounding system (GSS) for determining the ice shell and ocean thickness through acoustic monitoring of cracking events in the ice shell.

\section{Conclusions}

The science return possible from this mission is such that if life is present in Europa's ice at a level comparable to one of the most extreme and desolate of environments on Earth (Lake Vostok ice) then this mission could detect biosignatures in Europa's icy surface. The combination of detection methods, detection limits, and scales of observations provided by the model payload and mission concept combine to make this possible.

In the absence of any signs of life, this mission is also designed to generate an incredibly valuable dataset about the chemistry of Europa's ice shell, its putative ocean, and the geological, geophysical, and chemical context of this ocean world. Either of the above outcomes is of fundamental scientific value to understanding the prospects for life in the solar system, and our place in it.

The Europa Lander mission concept is mature, technologically well-developed, and ready to proceed to Phase A. 